\title{
A randomized phase II study of capecitabine-based chemoradiation with or without bevacizumab in resectable locally advanced rectal cancer: clinical and biological features
}

\author{
Ramon Salazar ${ }^{1 *}$, Jaume Capdevila², Berta Laquente', Jose Luis Manzano ${ }^{3}$, Carles Pericay ${ }^{4}$, Mercedes Martínez Villacampa', \\ Carlos López ${ }^{5}$, Ferran Losa ${ }^{6}$, Maria Jose Safont ${ }^{7}$, Auxiliadora Gómez ${ }^{8}$, Vicente Alonso ${ }^{9}$, Pilar Escudero ${ }^{10}$, \\ Javier Gallego ${ }^{11}$, Javier Sastre ${ }^{12}$, Cristina Grávalos ${ }^{13}$, Sebastiano Biondo ${ }^{14}$, Amalia Palacios ${ }^{15}$ and Enrique Aranda
}

\begin{abstract}
Background: Perioperatory chemoradiotherapy (CRT) improves local control and survival in patients with locally advanced rectal cancer (LARC). The objective of the current study was to evaluate the addition of bevacizumab (BEV) to preoperative capecitabine (CAP)-based CRT in LARC, and to explore biomarkers for downstaging.

Methods: Patients (pts) were randomized to receive 5 weeks of radiotherapy $45 \mathrm{~Gy} / 25$ fractions with concurrent CAP $825 \mathrm{mg} / \mathrm{m}^{2}$ twice daily 5 days per week and BEV $5 \mathrm{mg} / \mathrm{kg}$ once every 2 weeks (3 doses) (arm A), or the same schedule without BEV (arm B). The primary end point was pathologic complete response (ypCR: $\left.y p T_{0} N_{0}\right)$.
\end{abstract}

Results: Ninety pts were included in arm A (44) or arm B (46). Grade 3-4 treatment-related toxicity rates were 16\% and $13 \%$, respectively. All patients but one (arm A) proceeded to surgery. The ypCR rate was $16 \%$ in arm $A$ and $11 \%$ in arm B ( $p=0.54$ ). Fifty-nine percent vs $39 \%$ of pts achieved T-downstaging (arm A vs arm B; $p=0.04$ ). Serial samples for biomarker analyses were obtained for 50 out of 90 randomized pts (arm A/B: 22/28). Plasma angiopoietin-2 (Ang-2) levels decreased in arm A and increased in $\operatorname{arm~B~(~} p<0.05$ at all time points). Decrease in Ang-2 levels from baseline to day 57 was significantly associated with tumor downstaging ( $p=0.02$ ).

Conclusions: The addition of BEV to CAP-based preoperative CRT has shown to be feasible in LARC. The association between decreasing Ang-2 levels and tumor downstaging should be further validated in customized studies.

Trial registry: Clinicaltrials.gov identifier NCT01043484. Trial registration date: 12/30/2009.

Keywords: Bevacizumab, Chemoradiotherapy, Locally-advanced, Rectal cancer, Resectable

\section{Background}

Surgery is the mainstay of curative therapy for patients with rectal cancer confined to the bowel and regional lymph nodes. Nevertheless, rectal cancers have a high incidence of local failure. Recurrent pelvic disease is associated with significant morbidity and substantially shorter survival [1]. Perioperatory chemoradiotherapy (CRT) improves local control and survival in patients with locally advanced (T3-T4) rectal cancer (LARC) [2].

\footnotetext{
* Correspondence: ramonsalazar@iconcologia.net

${ }^{1}$ Catalan Institute of Oncology (ICO), Bellvitge Biomedical Research Institute (IDIBELL), L'Hospitalet de Llobregat, Barcelona, Spain

Full list of author information is available at the end of the article
}

Moreover, this strategy maximizes downstaging, increases the rate of sphincter-sparing surgery and provides early exposure to systemic therapy.

Capecitabine is being integrated into the treatment of patients with colorectal cancer as an alternative to 5fluorouracil (5-FU), resulting in improved convenience without compromising efficacy [3]. In addition, radiation induces thymidine phosphorylase and enhances the efficacy of capecitabine, leading to a synergistic effect [4]. A recent non-inferiority phase III study has shown capecitabine can replace 5-FU in adjuvant or neoadjuvant CRT regimens for patients with LARC [5]. 
In rectal cancer, several trials of bevacizumab with chemoradiation have shown promising results [6-11], but the lack of randomization and the bias associated with single-arm trials raises important concerns when interpreting these data. No randomized study to date has tested the use of bevacizumab in the neoadjuvant setting for localized disease.

Based on our previous experience (back to back submission [12]) and reports of preliminary feasibility of bevacizumab and chemoradiation in rectal cancer, we conducted a randomized trial of neoadjuvant bevacizumab and chemoradiation in patients with resectable LARC. Also, as little data exists on the role of biomarkers as predictors of response to bevacizumab when adding to preoperative CRT in LARC, we explored potential biomarkers that have been previously found to change in response to bevacizumab in other translational trials [11,13-20].

The following possible prognostic factors for tumor angiogenesis were evaluated: vascular endothelial growth factor (VEGF) and circulating soluble VEGF receptor 2 (VEGFR-2) which can be expressed in malignant tumors [21], angiopoietin-2 (Ang-2) expression, a molecule which promotes destabilization of blood vessels, and whose expression decreases along with microvessel density (MVD) after bevacizumab administration supporting the theory on the normalization of the vessels postulated for that drug [18], and the intratumor MVD since an increase in the number of tumor vessels might constitute a higher risk to develop metastasis [22-25].

\section{Methods}

This open, multicenter randomized phase II trial was carried out by the Spanish Cooperative Group for the Treatment of Digestive Tumors (TTD group). The study was conducted in accordance with the Declaration of Helsinki and Good Clinical Practice Guidelines. Before starting the study, written informed consent was obtained from all patients in the study. The protocol was approved by the institutional review boards of all participating centers. Reference Ethic Committee: Comité Ético de Investigación Clínica del Hospital Universitario 12 de Octubre, Avda de Córdoba, s/n, 28041 Madrid.

\section{Patient selection}

Patients 18 years of age or older with locally advanced rectal adenocarcinoma, clinical stage II-III [American Joint Committee on Cancer version 6: pelvic magnetic resonance imaging (MRI) was used to define $\mathrm{T}$ category and $\mathrm{N}$ category], within $<15 \mathrm{~cm}$ from the anal verge, and an Eastern Cooperative Oncology Group (ECOG) performance status of 0 or 1 were eligible. All patients were required to be candidates for definitive surgical resection. Patients had adequate bone marrow and organ function and no previous chemotherapy or radiation for rectal cancer. Exclusion criteria included uncontrolled hypertension, clinically significant cardiac disease, having undergone major surgery within $28 \mathrm{~d}$ of trial therapy, recent or current use of fulldose oral or parenteral anticoagulants or thrombolytic agents, chronic daily treatment with high-dose aspirin, or treatment with nonsteroidal anti-inflammatory drugs.

\section{Treatment schedule}

Patients were randomly allocated in a 1:1 ratio to CRT treatment with or without bevacizumab, using permuted blocks with stratification by center and tumor location (upper or middle third $v s$ lower third). Radiotherapy (RT) consisted of a total of 45 Gy delivered in 25 daily fractions over 5 weeks (1.8 Gy/d for $5 \mathrm{~d} / \mathrm{wk}$ ). Patients in arm A received concomitant bevacizumab $(5 \mathrm{mg} / \mathrm{kg})$ on day 1 of weeks 1,3 , and 5 , plus capecitabine $\left(825 \mathrm{mg} / \mathrm{m}^{2}\right)$ twice daily concomitant with RT; the same schedule without bevacizumab was administered to patients in arm B. One cycle was considered two weeks for cycle 1 and 2, and one week for cycle 3 . Standard surgery, , was performed 6-8 weeks after the completion of CRT. A radical resection of the rectal tumor along with an appropriate vascular pedicle and accompanying lymphatic drainage was made. For tumors in the mid and lower rectum total mesorectal excision (TME) was carried out. However, for tumors in the upper rectum (at or above $10 \mathrm{~cm}$ from the anal margin) the mesorectum was resected at $5 \mathrm{~cm}$ or more distal to the tumor. Postoperative adjuvant chemotherapy was administered at the investigators discretion.

The protocol stipulated detailed capecitabine dosemodification criteria according to toxicity, graded using the National Cancer Institute Common Toxicity Criteria (NCI-CTC) version 3.0. No dose reductions for bevacizumab were planned.

\section{Evaluations during the study}

Pretreatment evaluation included a complete medical history and physical examination, hematology with differential leucocyte count, chemistry, coagulation profile, urinalysis, carcinoembryonic antigen, electrocardiogram, complete colonoscopy with biopsy, abdominal and thoracic computerized axial tomography (or thoracic x-ray), and pelvic MRI. Medical history, physical examination, and laboratory studies were repeated prior to the start of each treatment cycle (days 1,15 and $29 \pm 2$ days). After surgery, histologic tumor infiltration (ypTypN) and grading of regression [assessed using the Mandard scale [26]] were evaluated.

All patients were scheduled for a follow-up period of 5 years after surgery.

\section{Biologics evaluation}

Participation in the biologic sub-study was optional. To those patients who gave their consent to have the 
biomarkers analyzed, plasma levels of VEGF, VEGFR-2, and Ang-2 were measured at baseline (d1, pre-treatment), 15 (d15) and 57 days (d57, post-treatment) after first treatment infusion by enzyme-linked immunosorbent assay (ELISA): Quantikine Immunoassays (R\&D Systems) were used according to the manufacturer's instructions.

Additionally, tissue samples (baseline and at surgery) were assessed for MVD by immunohistochemistry, as described previously [27].

\section{Statistical analysis}

The primary end-point was pathological complete response (pCR), as defined by $\mathrm{yp}_{0} \mathrm{~N}_{0}$. Based on previous trials, a conservative estimate of $\mathrm{pCR}$ rate for patients with LARC treated with capecitabine and radiation is approximately 15\% [28-35]. Following the SWE method for randomized phase II clinical trials [36], assuming a minimum pCR rate of at least $15 \%$ in one of the arms, a difference between the two arms of $10 \%$, and accepting a probability of correct selection of $87 \%, 41$ pts per arm were needed. Considering a $10 \%$ of non-evaluable patients, the study needed to enroll a minimum of 90 patients.

The primary efficacy analyses were conducted on an intention to treat (ITT) basis (all randomized patients). The safety analysis was performed for the safety population (patients who initiated trial therapy). The statistical analyses were performed using SAS version 9.2.

The pCR rate was calculated and the $95 \%$ confidence intervals (CI) were estimated using normal approximation.
Secondary end-points were safety, and rates of downstaging (lower ypT compared with the pretreatment clinical T), sphinctersparing surgery, local recurrence, post-surgical complication and of complete resection (R0). Proportions were compared using a $x^{2}$ test or, if this could not be used, a Fisher's exact test.

All statistical tests were two-sided. The significance level was established at a value of $\alpha=0.05$.

The intra-individual differences at different time points for the concentrations of biomarkers were tested through paired t-Test if the population was normally distributed, or else through Wilcoxon signed rank test. To test these differences between groups of treatment, Student test was used if the distribution was normal, and Mann-Whitney test if it was not normally distributed.

Logistic regression model was adopted to estimate and test the biomarkers for their association with downstaging. Results were expressed as odds ratios and their 95\% CI. Data analysis is reported according to REMARK guidelines [37].

\section{Results}

Between December 2009 and March 2011, 90 patients were randomly assigned through 12 Spanish hospitals, 44 in arm A and 46 in arm B (Figure 1).

Baseline patient characteristics were well balanced between groups (Table 1). The median distance from anal verge was $6.5 \mathrm{~cm}$ in arm $A$ and $7.0 \mathrm{~cm}$ in arm $B$.

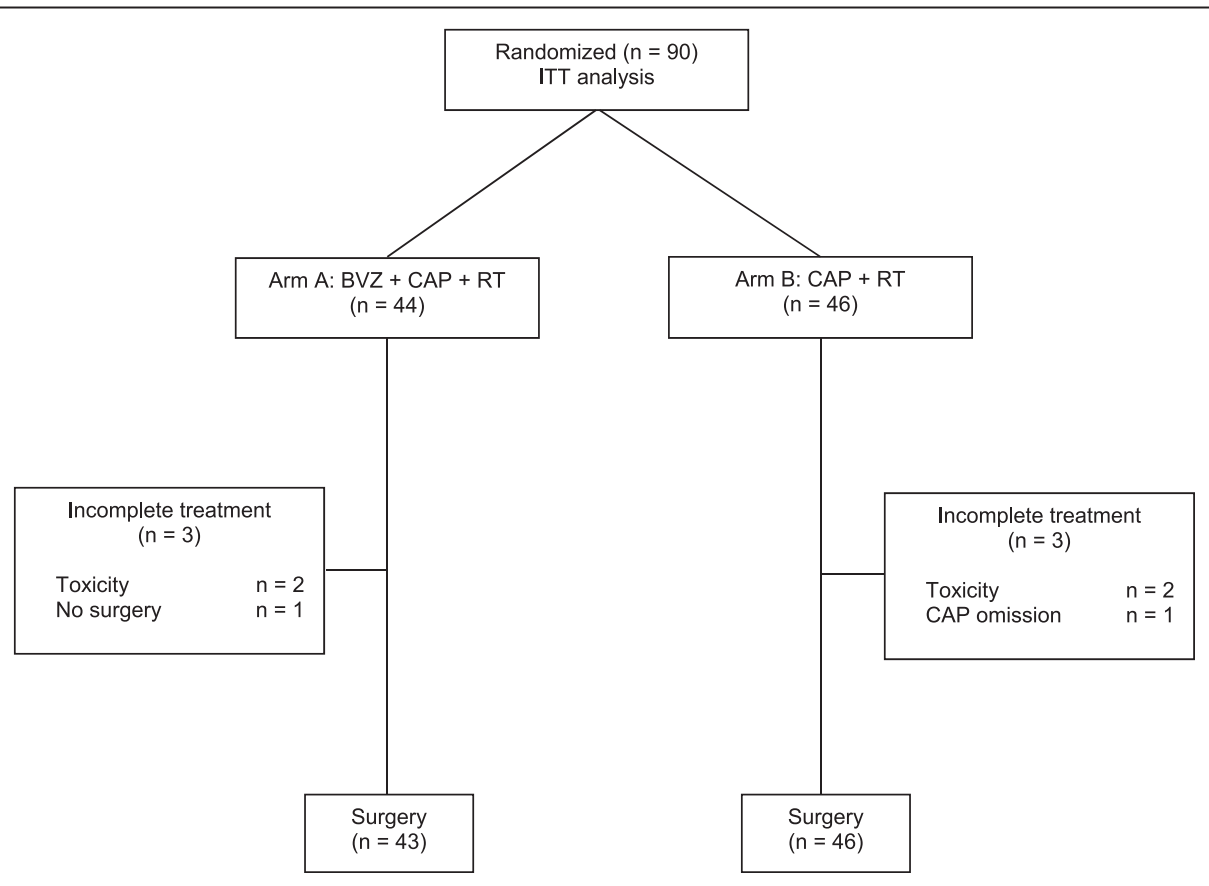

Figure 1 CONSORT diagram. 
Table 1 Baseline characteristics: intention to treat population $(n=90)$

\begin{tabular}{|c|c|c|c|c|}
\hline \multirow[b]{2}{*}{ Parameter } & \multicolumn{2}{|c|}{$\begin{array}{l}\text { Arm A } \\
(B V Z+C A P+R T) \\
\left(n^{\circ} \text { patients }=44\right)\end{array}$} & \multicolumn{2}{|c|}{$\begin{array}{l}\text { Arm B } \\
(C A P+R T) \\
\left(n^{\circ} \text { patients }=46\right)\end{array}$} \\
\hline & No. of patients & $\%$ & No. of patients & $\%$ \\
\hline \multicolumn{5}{|l|}{ Sex } \\
\hline Male & 25 & 57 & 30 & 65 \\
\hline Female & 19 & 43 & 16 & 35 \\
\hline \multicolumn{5}{|l|}{ Age, years } \\
\hline Median & 64 & & 60 & \\
\hline Range & $37-77$ & & $42-78$ & \\
\hline \multicolumn{5}{|l|}{ ECOG } \\
\hline 0 & 22 & 50 & 30 & 65 \\
\hline 1 & 22 & 50 & 16 & 35 \\
\hline \multicolumn{5}{|l|}{ Tumor location } \\
\hline Upper third & 10 & 23 & 10 & 22 \\
\hline Middle third & 14 & 32 & 19 & 41 \\
\hline Lower third & 20 & 45 & 16 & 35 \\
\hline Unknown & 0 & 0 & 1 & 2 \\
\hline \multicolumn{5}{|c|}{ Clinical tumor category } \\
\hline $\mathrm{T}_{2}$ & 1 & 2 & 1 & 2 \\
\hline $\mathrm{T}_{3}$ & 33 & 75 & 38 & 83 \\
\hline $\mathrm{T}_{4}$ & 10 & 23 & 7 & 15 \\
\hline \multicolumn{5}{|c|}{ Clinical nodal category } \\
\hline $\mathrm{N}_{0}$ & 7 & 16 & 5 & 11 \\
\hline$N_{1}$ & 18 & 41 & 27 & 59 \\
\hline $\mathrm{N}_{2}$ & 19 & 43 & 14 & 30 \\
\hline \multicolumn{5}{|l|}{ Clinical TNM } \\
\hline$\| \mathrm{A}$ & 6 & 14 & 5 & 11 \\
\hline$\| \mathrm{B}$ & 1 & 2 & 0 & 0 \\
\hline$\| \mathrm{IIB}$ & 18 & 41 & 27 & 59 \\
\hline$\| I I C$ & 19 & 43 & 14 & 30 \\
\hline
\end{tabular}

\section{Treatment compliance}

Treatment compliance was similar in both arms. Fortyone $(93 \%)$ and forty-three (93\%) patients completed the planned CRT treatment in arm A and arm B, respectively.

Five patients received a dose of RT of lower than $45 \mathrm{~Gy}, 3$ in arm A ( 1 of them discontinued the treatment after receiving 30.6 Gy, due to toxicity) and 2 in arm B. Two patients in arm $A$ and three patients in arm $B$ received $<3$ cycles of capecitabine, due to toxicity; a dose reduction of capecitabine was performed in 1 patient (toxicity). In arm A, all but one patient (toxicity) received the planned 3 cycles of bevacizumab.

\section{Safety}

Treatment-related toxicity occurring at a frequency $>10 \%$ of patients is summarized in Table 2. The overall rate of
Table 2 Early adverse events related ${ }^{a}$ to treatment $(\geq 10 \%)$ per patient (\%) according to $\mathrm{NCl}-\mathrm{CTC}$ criteria v3.0

\begin{tabular}{|c|c|c|c|c|}
\hline & \multicolumn{2}{|c|}{$\begin{array}{l}\text { Arm A } \\
(B V Z+C A P+R T) \\
\left(n^{\circ} \text { patients }=44\right)\end{array}$} & \multicolumn{2}{|c|}{$\begin{array}{l}\text { Arm B } \\
(C A P+R T) \\
\left(n^{\circ} \text { patients }=46\right)\end{array}$} \\
\hline & $\%$ grade $1 / 2$ & $\%$ grade 3 & $\%$ grade $1 / 2$ & $\%$ grade 3 \\
\hline Astenia/fatigue & 55 & 2 & 24 & - \\
\hline Diarrhea & 36 & - & 41 & - \\
\hline Dysuria & 18 & - & 30 & - \\
\hline Rectal tenesmus & 18 & - & 17 & - \\
\hline Nausea & 13 & - & 11 & - \\
\hline $\begin{array}{l}\text { Hand-foot } \\
\text { syndrome }\end{array}$ & 14 & - & 4 & 2 \\
\hline $\begin{array}{l}\text { Anorectal } \\
\text { discomfort }\end{array}$ & 9 & 2 & 9 & - \\
\hline Anorexia & 9 & - & 11 & - \\
\hline
\end{tabular}

Note: there were no grade 4 events.

${ }^{a}$ An adverse event was considered attributable to bevacizumab, capecitabine or radiation if it was deemed remotely, possibly or probably related.

patients with grade 3 to 4 treatment-related toxicity was $16 \%$ in arm A versus $13 \%$ in arm B $(p=0.70)$. There was no grade 3 or greater hematological toxicity. Three patients in arm A (grade $\leq 2$ ) and two in arm B (one grade 1 and another grade 3 ) had hypertension, two of them considered as probably related to the study treatment ( $\operatorname{arm~A})$. Surgery was performed after a median interval of 51 days (range, 36-100 days). All included patients but one (arm A: peritoneal carcinomatosis) proceeded to surgery. Anterior resection and abdominoperineal resection were performed in $27(61 \%)$ and 15 patients (34\%) in arm A and in 31 (67\%) and 13 patients (28\%) in arm B, respectively: other procedures were performed in the remaining patients. Thirty-four (77\%) patients in arm A and 36 (78\%) in arm B underwent TME: the remaining patients undergoing surgery (9 in arm A and 10 in arm B) had a partial mesorectal excision (PME) due to their tumors were located at the upper rectum. Sphincter preservation was achieved in 27 (61\%) and 31 (67\%) patients in arm A and B, respectively. The overall rate of surgical complications was not significantly different between groups. Two patients in arm A and 6 patients in arm B experienced local complications. Nineteen patients (43\%) and $18(39 \%)$ patients in arm A and $\mathrm{B}$ experienced at least one postoperative complication, respectively. Ten patients (7 in arm A (15.9\%) and 3 in arm B (6.5\%)) required reoperation, due to anastomotic dehiscence. There were no perioperative deaths. During surgery, distant metastases in abdomen were found in 4 patients, all of them in arm B.

\section{Response to treatment}

The ypCR $\left(\mathrm{ypT}_{0} \mathrm{~N}_{0}\right)$ rate in the ITT population was $16 \%$ (7/44 patients; 95\% CI 7-31\%) in arm A and 11\% (5/46 
patients; 95\% CI 4-24\%) in arm B ( $p=0.54)$. Absence of residual tumor (R0) was achieved in all except 3 patients, who had microscopic residual disease (R1). Sixteen (36\%) patients in arm A and $20(44 \%)$ patients in arm B attained Mandard tumor regression grade (TRG) 1 or 2 responses. A decrease in the $\mathrm{T}$ stage ( $\mathrm{T}$ downstaging) was achieved by 26 (59\%) of 43 operated patients in arm A versus $18(39 \%)$ of 46 in arm B $(p=0.04)$ (Table 3).

After a median follow-up of 18 months (range, 3-28 months), 88 (98\%) patients remained alive, and 73 (81\%) patients continued to be free of any sign of disease. One patient in arm B had a local recurrence, six patients in each arm developed distant metastases, four patients in arm B presented a second tumor (one of them was the patient with local recurrence), and two patients in arm A had died due to the underlying cancer (one of them had presented distant metastases).

\section{Study of prognostic factors}

At the time of analysis, biomarker outcome data were available for 50 of the 90 randomized patients $(56 \%$ of trial participants), 22/44 (50\%) treated in arm A and 28/ $46(61 \%)$ in arm B. At least paired plasma samples were

Table $3 \mathbf{T}$ and $\mathbf{N}$ downstaging (intention to treat population: 90 patients)

\begin{tabular}{|c|c|c|c|c|c|}
\hline \multirow[b]{2}{*}{ Parameter } & \multicolumn{2}{|c|}{$\begin{array}{l}\text { Arm A } \\
(B V Z+C A P+R T) \\
\left(n^{\circ} \text { patients }=44\right)\end{array}$} & \multicolumn{2}{|c|}{$\begin{array}{l}\text { Arm B } \\
(C A P+R T) \\
\left(n^{\circ} \text { patients }=46\right)\end{array}$} & \multirow[t]{2}{*}{$p$ value } \\
\hline & $\begin{array}{l}\text { No. of } \\
\text { patients }\end{array}$ & $\%$ & $\begin{array}{l}\text { No. of } \\
\text { patients }\end{array}$ & $\%$ & \\
\hline ypT & & & & & 0.0429 \\
\hline Better & 26 & 59 & 18 & 39 & \\
\hline Equal & 16 & 37 & 28 & 61 & \\
\hline Worse & 1 & 2 & 0 & 0 & \\
\hline Missing $^{\mathrm{a}}$ & 1 & 2 & 0 & 0 & \\
\hline ypN & & & & & 0.0865 \\
\hline Better & 24 & 55 & 35 & 76 & \\
\hline Equal & 15 & 34 & 8 & 18 & \\
\hline Worse & 4 & 9 & 2 & 4 & \\
\hline No Evaluable ${ }^{\mathrm{b}}$ & 0 & 0 & 1 & 2 & \\
\hline Missing $^{a}$ & 1 & 2 & 0 & 0 & \\
\hline ypT and ypN & & & & & 0.5612 \\
\hline Better in both & 18 & 41 & 16 & 35 & \\
\hline $\begin{array}{l}\text { Better in one of } \\
\text { them }\end{array}$ & 14 & 32 & 20 & 43 & \\
\hline Worse in both & 11 & 25 & 9 & 20 & \\
\hline No Evaluable ${ }^{b}$ & 0 & 0 & 1 & 2 & \\
\hline Missing $^{a}$ & 1 & 2 & 0 & 0 & \\
\hline
\end{tabular}

available for 18 patients in arm A and 23 in arm B; tumor samples (pretreatment and from surgical specimen) were available from 12 and 18 patients in arm A and B, respectively (Figure 2).

Downstaging was evaluated in 49 out of the $50 \mathrm{pa}$ tients included in the sub-study: one patient did not undergo surgery. Eleven patients in each arm were downstaged: no statistically significant differences were observed between groups ( $p=0.36$ ).

No differences were observed in baseline levels of any biomarker between both arms. The pretreatment level of biomarkers showed no association with downstaging.

Angiopoietin-2 levels were significantly higher in arm B than in arm $\mathrm{A}$ at $\mathrm{d} 15(p=0.0056)$ and $\mathrm{d} 57(p=0.0133)$ (Table 4). Angiopoietin-2 levels significantly decreased in arm A at $\mathrm{d} 15(p=0.04)$ and increased in arm B $(p=0.01)$ at $\mathrm{d} 57$. When intergroup differences were studied, plasma Ang-2 levels decreased in arm A and increased in arm B at all time points compared to baseline level, with significant differences between levels in group A and B at all time points. Overall, decrease in Ang-2 levels from baseline to d57, was significantly associated with tumor downstaging (OR: 0.95, 95\% CI 0.91-0.99; $p=0.02$ ).

In contrast to serum Ang-2, VEGF levels increased in arm $\mathrm{A}$ and decreased in arm B at all time points compared to baseline levels, with significant intergroup differences at all time points. Nevertheless, there was no significant association between serum VEGF levels and downstaging.

There were no significant changes in other biomarker levels and none was associated with tumor downstaging.

\section{Discussion}

This study confirms the feasibility of preoperative CRT with bevacizumab and capecitabine in patients with LARC, in a randomized trial. Although the patients who received bevacizumab tended to have a higher $\mathrm{pCR}$ rate, the predefined efficacy endpoint was not met. Interesting, Ang-2 plasma levels significantly decreased along the study in patients receiving bevacizumab and, overall, decrease in Ang-2 levels was significantly associated with tumor downstaging: those findings suggest improved tumor shrinkage related to the use of bevacizumab and a potential role of plasma Ang-2 to monitor downstaging.

We conducted this study to further understand the role of bevacizumab in the treatment of LACR. The $5 \mathrm{mg} / \mathrm{kg}$ dose was chosen based on the toxicity observed in the Willet et al. study [18], and the Xeberecto Trial (back to back submission [12]), a phase II study of preoperative bevacizumab, capecitabine and radiotherapy for resectable LARC. Our trial was initially designed to include oxaliplatin in both arms; however because the results of two large randomized studies [38,39] did not demonstrate the benefit of oxaliplatin with concurrent 


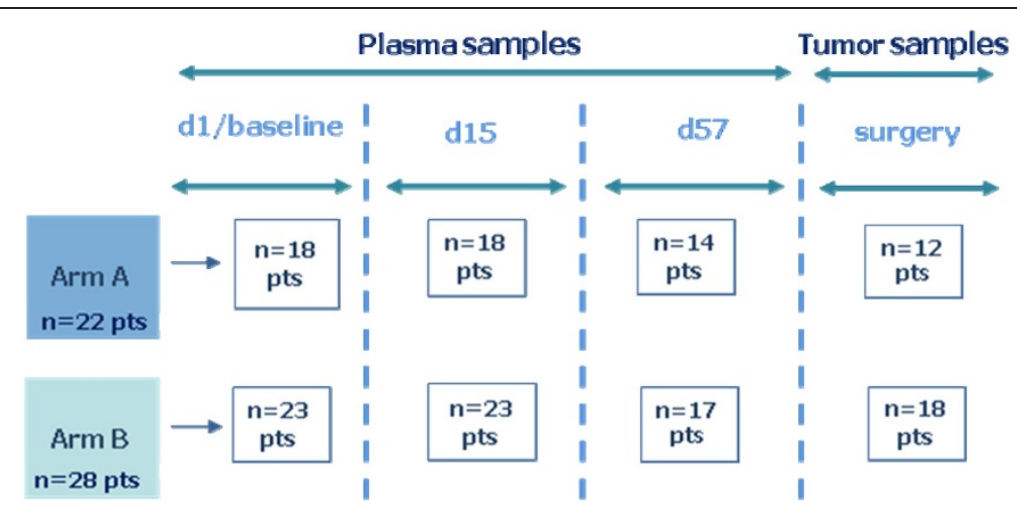

Figure 2 Plasma and tumor samples availability for biomarker analyses.

irradiation, the study was amended to do not use this drug.

Our patients had a relatively high risk for pelvic recurrence $(98 \%$ were $\mathrm{T} 3$ or $\mathrm{T} 4$, and $87 \%$ were $\mathrm{N}+$ ). The reached $\mathrm{pCR}$ rate of $16 \%$, albeit not too high, is within the range (13-36\%) reported across a number of phase II studies evaluating bevacizumab plus CRT (Table 5) [6-11]. However, caution is needed when comparing pCR rates as this item itself is highly dependent on the quality of the pathological examination [40].

The decrease in the $\mathrm{T}$ stage was significantly higher in patients receiving bevacizumab, although the rate of downstaging in both arms was lower than in other single arm studies using preoperative bevacizumab plus CRT $[6,10,11]$. This should be interpreted with caution because of the preoperative MRI staging technical limitation and lack of central imaging evaluation.
Toxicities were expected and manageable. Early toxicity was mild in both arms. The most serious post-operative complication was anastomotic dehiscence, which occurred more frequently in patients treated with bevacizumab, but in the range reported by others [6,7,9-11].

Suitable biomarkers predicting patients who are likely to benefit from bevacizumab treatment remain elusive [41]. In our exploratory analyses decrease in Ang-2 levels from baseline to d57 was significantly associated with tumor downstaging. Moreover, Ang-2 levels decreased in the bevacizumab arm and increased in the other arm at all time points compared to baseline levels, with significant differences between levels in both groups at all time points. Angiopoietin-2 has been proposed as a gatekeeper of VEGF function and vascular remodeling [42,43], and has been shown to promote metastatic growth [44]. Goede et al. [14] found that serum levels of Ang-2 in patients

Table 4 Evolution of biomarkers during the study compared to pretreatment (basal) value

\begin{tabular}{|c|c|c|c|c|c|c|c|c|}
\hline & \multicolumn{4}{|c|}{$\begin{array}{l}\text { Arm A } \\
(B V Z+C A P+R T)\end{array}$} & \multicolumn{4}{|c|}{$\begin{array}{l}\text { Arm B } \\
(C A P+R T)\end{array}$} \\
\hline & VEGF & VEGFR-2 & Ang-2 & $M V D^{\mathbf{b}}$ & VEGF & VEGFR-2 & Ang-2 & $M V D^{\mathbf{b}}$ \\
\hline \multicolumn{9}{|l|}{ Basal } \\
\hline$n$ & 18 & 18 & 18 & 12 & 23 & 23 & 23 & 18 \\
\hline Median & 56 & 9056 & 2424 & 14 & 85 & 9282 & 3609 & 14 \\
\hline$p$ & NA & NA & NA & NA & NA & NA & NA & NA \\
\hline \multicolumn{9}{|l|}{$\mathrm{D} 15^{\mathrm{a}}$} \\
\hline$n$ & 18 & 18 & 18 & 12 & 23 & 23 & 23 & 18 \\
\hline Median & 13 & 7 & -22 & -8 & -40 & 4 & 16 & 34 \\
\hline$p$ & 0.19 & 0.18 & 0.04 & 0.73 & 0.11 & 0.33 & 0.11 & 0.03 \\
\hline \multicolumn{9}{|l|}{$\mathrm{D} 57^{\mathrm{a}}$} \\
\hline$n$ & 14 & 14 & 13 & NA & 17 & 17 & 17 & NA \\
\hline Median & 55 & 7 & -16 & NA & -13 & -2 & 20 & NA \\
\hline$p$ & 0.03 & 0.20 & 0.20 & NA & 0.71 & 0.86 & 0.01 & NA \\
\hline
\end{tabular}

Abbreviations: IQR interquartile range, VEGF vascular endothelial growth factor, VEGFR-2 circulating soluble VEGF receptor 2, Ang-2 angiopoietin-2.

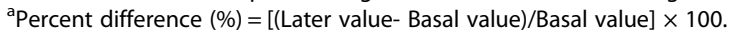

${ }^{\mathrm{b}}$ Tissue samples (baseline and at surgery). 
Table 5 Clinical trials of bevacizumab + radiochemotherapy as pre-operative treatment of locally advanced rectal cancer

\begin{tabular}{|c|c|c|c|c|}
\hline Author and regimen & No. of patients & pCR (yp To- $\left.\mathrm{N}_{0} ; \%\right)$ & T-downstaging (\%) & Grade 3 most common toxicities \\
\hline \multicolumn{5}{|l|}{ Willet [11] } \\
\hline $\mathrm{BVZ}+5 \mathrm{FU}+\mathrm{RT}$ & 32 & $16^{\mathrm{a}}$ & 50 & Diarrhea and hypertension \\
\hline \multicolumn{5}{|l|}{ Spigel [9] } \\
\hline$B V Z+5 F U+R T$ & 35 & 29 & NA & Diarrhea \\
\hline \multicolumn{5}{|l|}{ Crane [6] } \\
\hline$B V Z+C A P+R T$ & 25 & 32 & 64 & Perianal desquamation \\
\hline \multicolumn{5}{|l|}{ Velenik [10] } \\
\hline$B V Z+C A P+R T$ & 61 & 13 & 45 & Dermatitis \\
\hline \multicolumn{5}{|l|}{ Kennecke [7] } \\
\hline $\mathrm{BVZ}+\mathrm{CAP}+\mathrm{OX}+\mathrm{RT}$ & 42 & 18 & NA & Diarrhea \\
\hline \multicolumn{5}{|l|}{ Nogue [8] } \\
\hline $\mathrm{BVZ}+\mathrm{XELOX} \rightarrow \mathrm{BVZ}+\mathrm{CAP}+\mathrm{RT}$ & 47 & 36 & NA & Rectal tenesmus \\
\hline \multicolumn{5}{|l|}{ Salazar (current study) } \\
\hline $\mathrm{BVZ}+\mathrm{CAP}+\mathrm{RT}$ & 44 & 16 & 26 & Astenia/fatigue \\
\hline$C A P+R T$ & 46 & 11 & 18 & \\
\hline
\end{tabular}

Abbreviations: $p C R$ pathological complete response, BVZ bevacizumab, 5FU 5-fluorouracil, CAP capecitabine, OX oxaliplatin, XELOX capecitabine + oxaliplatin, $R T$ radiotherapy, $N A$ not available.

${ }^{\mathrm{a}} \mathrm{ypT}_{0 .}$.

with metastatic colorectal cancer were significantly higher than in healthy individuals: moreover, in that study, compared with high serum Ang-2 levels, low serum Ang-2 was associated with an outstanding response rate, better disease control and excellent overall survival (OS). Although one can only speculate about this relationship, it seems plausible that adding bevacizumab to standard CRT induces a decrease in serum Ang-2 levels that could facilitate tumor regression.

On the contrary, we found that serum VEGF levels increased in arm A and decreased in arm B at all time points compared to baseline levels, with significant intergroup differences at all time points: this finding had not influence on downstaging. Similarly, several studies have shown acute increases in circulating VEGF after the start of bevacizumab [11]. Nevertheless, changes in VEGF concentrations associated with bevacizumab treatment have not necessarily been predictive of benefit [41]. Detailed analyses are needed of total and free VEGF levels during treatment before circulating VEGF is dismissed as a biomarker.

In accordance with previous reports $[11,12,14,45]$, pretreatment level of VEGF and tumor MVD was not correlated to clinical end points.

This trial has several limitations. Firstly, the selection of patients for the biologic sub-study was opportunistic, by including those patients who gave their consent and in which assessment of selected biomarkers was available at different points, although there is no reason to suspect any differences with other patients where those determinations were not possible. Secondly, the small size of each cohort in that study makes it difficult to find potential associations between changes in different biomarkers, and comparisons between both arms: nevertheless, our findings point in the same direction as that described by other authors. Thirdly, TME was not possible in around $20 \%$ of patients, due to the tumor localization: as stated previously [5], TME was mandatory for tumors in the lower two-thirds of the rectum, with PME being permitted for those in the upper third, provided a distal margin of at least $5 \mathrm{~cm}$ without coning was observed. Finally, as in other studies realized in patients with LARC, 4 patients were found having distant metastases at the time of surgery, a number not very different of those published by others [39]. As we didn't make a reevaluation of the baseline studies, we cannot discharge a possible mistake in the inclusion of any patient.

\section{Conclusions}

The results of this randomized study support the data described previously in single arm studies about the feasibility of the addition of bevacizumab to a standard neoadjuvant capecitabine-based CRT regimen, as well as its potential role in downstaging. It will be also important to continue observation of these patients to elucidate long-term outcome and morbidity of this strategy. Furthermore, although definitive judgment on the role of Ang-2 as a specific biomarker of outcome to bevacizumab 
in LARC will require further analysis of larger numbers of patients from phase III trials, the results arising from this study should encourage researchers to further investigate the value of Ang-2 amongst others as a potential biomarker to monitor the added value of bevacizumab in clinically relevant endpoints.

\begin{abstract}
Abbreviations
5-FU: 5-fluorouracil; Ang-2: Angiopoietin-2; BEV: Bevacizumab; CAP: Capecitabina; CRT: Chemoradiotherapy; ECOG: Eastern Cooperative Oncology Group; ELISA: Enzyme-linked immunosorbent assay; ITT: Intention to treat; LARC: Locally advanced rectal cancer; MRI: Magnetic resonance imaging; MVD: Microvessel density; NCI-CTC: National Cancer Institute Common Toxicity Criteria; OS: Overall survival; pCR: Pathological complete response; pts: Patients; RO: Radical resection; RT: Radiotherapy; TME: Total mesorectal excision; TRG: Tumor regression grade; TTD group: Spanish Cooperative Group for the Treatment of Digestive Tumors; VEGF: Vascular endothelial growth factor; ypCR: Pathologic complete response.
\end{abstract}

\section{Competing interests}

Enrique Aranda, Consultant or Advisory Role: Roche and Merck Serono. All remaining authors declare that they have no competing interests.

\section{Authors' contributions}

RS was responsible for conception and design, data analysis and interpretation, and manuscript writing; CG and BL were responsible for conception and design and data analysis and interpretation; SB and AP were responsible for conception and design. RS, JC, BL, JLM, CP, MMV, CL, FL, MJS, AG, VA, PE, JG, JS, $C G, S B, A P$ and $E A$ were responsible for provision of study materials or patients, collection and assembly of data, and final approval of manuscript.

\section{Acknowledgements}

The authors thank the patients and the medical and nursing staff of all the participating institutions.

Ramón Salazar, Mercedes Martínez Villacampa and Berta Laquente (Instituto Catalán de Oncología); Cristina Grávalos (Hospital 12 de Octubre); Enrique Aranda, Amalia Palacios and Auxiliadora Goméz (Hospital Universitario Reina Sofía); Sebastián Biondo (Hospital Universitario de Bellvitge); Jaume Capdevila (Hospital Universitari Vall D’Hebrón); José Luis Manzano (Hospital U. German Trias I Pujol); Carles Pericay (Complejo Sanitario Parc Tauli); Carlos López (Hospital U. Marqués de Valdecilla); Ferran Losa Gaspa (Hospital G. de L'Hospitalet); Maria José Safont (Hospital General U. de Valencia); Vicente Alonso (Hospital Universitario Miguel Servet); Pilar Escudero (Hospital Clínico U. Lozano Blesa); Javier Gallego Plazas (Hospital General U. de Elche); Javier Sastre (Hospital C. U. San Carlos).

TTD Data Center: Inma Ruiz de Mena

Monitoring, Statistics and Data Management: Pivotal

Financial support for this research trial was provided by Roche Farma, S.A.

Support for third-party writing assistance for this manuscript was provided by Roche.

Supported by the TTD, Madrid, Spain.

\section{Funding}

Financial support for this research trial was provided by Roche Farma, S.A.

\section{Author details}

${ }^{1}$ Catalan Institute of Oncology (ICO), Bellvitge Biomedical Research Institute (IDIBELL), L'Hospitalet de Llobregat, Barcelona, Spain. ${ }^{2}$ Medical Oncology, Hospital Universitari Vall D'Hebrón, Barcelona, Spain. ${ }^{3}$ Medical Oncology, Hospital Universitari German Trias I Pujol, Barcelona, Spain. ${ }^{4}$ Medical Oncology, Complejo Sanitario Parc Taulí, Barcelona, Spain. ${ }^{5}$ Medical Oncology, Hospital Universitario Marqués de Valdecilla, Santander, Spain. ${ }^{6}$ Medical Oncology, Hospital General de L'Hospitalet, Barcelona, Spain. ${ }^{7}$ Medical Oncology, Hospital General Universitario de Valencia, Valencia, Spain. ${ }^{8}$ Medical Oncology, Reina Sofía Hospital, University of Córdoba, Maimonides Institute of Biomedical Research (IMIBIC); Spanish Cancer Network (RTICC), Instituto de Salud Carlos III, Córdoba, Spain. ${ }^{9}$ Medical Oncology, Hospital Universitario Miguel Servet, Zaragoza, Spain. ${ }^{10}$ Medical Oncology, Hospital Clínico Universitario Lozano Blesa, Zaragoza, Spain. ${ }^{11}$ Medical Oncology, Hospital General U. de Elche, Alicante, Spain. ${ }^{12}$ Medical
Oncology, Hospital Clínico Universitario San Carlos, Madrid, Spain. ${ }^{13}$ Medical Oncology, Hospital Doce de Octubre, Madrid, Spain. ${ }^{14}$ General and Digestive Surgery Hospital Universitario de Bellvitge, Barcelona, Spain. ${ }^{15}$ Radiation Oncology, Hospital Universitario Reina Sofía, Córdoba, Spain.

Received: 7 April 2014 Accepted: 29 January 2015

Published online: 26 February 2015

\section{References}

1. Braendengen M, Tveit KM, Berglund A, Birkemeyer E, Frykholm G, Påhlman L, et al. Randomized phase III study comparing preoperative radiotherapy with chemoradiotherapy in nonresectable rectal cancer. J Clin Oncol Off J Am Soc Clin Oncol. 2008;26:3687-94.

2. Sauer R, Becker H, Hohenberger W, Rödel C, Wittekind C, Fietkau R, et al. Preoperative versus postoperative chemoradiotherapy for rectal cancer. N Engl J Med. 2004;351:1731-40.

3. Twelves C, Gollins S, Grieve R, Samuel L. A randomised cross-over trial comparing patient preference for oral capecitabine and 5-fluorouracil/ leucovorin regimens in patients with advanced colorectal cancer. Ann Oncol Off J Eur Soc Med Oncol ESMO. 2006;17:239-45.

4. Kocakova I, Svoboda M, Kubosova K, Chrenko V, Roubalova E, Krejci E, et al. Preoperative radiotherapy and concomitant capecitabine treatment induce thymidylate synthase and thymidine phosphorylase mRNAs in rectal carcinoma. Neoplasma. 2007;54:447-53.

5. Hofheinz R-D, Wenz F, Post S, Matzdorff A, Laechelt S, Hartmann JT, et al. Chemoradiotherapy with capecitabine versus fluorouracil for locally advanced rectal cancer: a randomised, multicentre, non-inferiority, phase 3 trial. Lancet Oncol. 2012;13:579-88.

6. Crane CH, Eng C, Feig BW, Das P, Skibber JM, Chang GJ, et al. Phase II trial of neoadjuvant bevacizumab, capecitabine, and radiotherapy for locally advanced rectal cancer. Int J Radiat Oncol Biol Phys. 2010;76:824-30.

7. Kennecke H, Berry S, Wong R, Zhou C, Tankel K, Easaw J, et al. Pre-operative bevacizumab, capecitabine, oxaliplatin and radiation among patients with locally advanced or low rectal cancer: a phase II trial. Eur J Cancer Oxf Engl 1990. 2012:48:37-45.

8. Nogué M, Salud A, Vicente P, Arriví A, Roca JM, Losa F, et al. Addition of bevacizumab to XELOX induction therapy plus concomitant capecitabine-based chemoradiotherapy in magnetic resonance imaging-defined poor-prognosis locally advanced rectal cancer: the AVACROSS study. The oncologist. 2011;16:614-20

9. Spigel DR, Bendell JC, McCleod M, Shipley DL, Arrowsmith E, Barnes EK, et al. Phase II study of bevacizumab and chemoradiation in the preoperative or adjuvant treatment of patients with stage $\|/ /\|$ rectal cancer. Clin Colorectal Cancer. 2012;11:45-52.

10. Velenik V, Ocvirk J, Music M, Bracko M, Anderluh F, Oblak I, et al. Neoadjuvant capecitabine, radiotherapy, and bevacizumab (CRAB) in locally advanced rectal cancer: results of an open-label phase II study. Radiat Oncol Lond Engl. 2011;6:105.

11. Willett CG, Duda DG, di Tomaso E, Boucher Y, Ancukiewicz M, Sahani DV, et al. Efficacy, safety, and biomarkers of neoadjuvant bevacizumab, radiation therapy, and fluorouracil in rectal cancer: a multidisciplinary phase II study. J Clin Oncol Off J Am Soc Clin Oncol. 2009;27:3020-6.

12. García M, Martinez-Villacampa M, Santos C, Navarro V, Teule A, Losa F, et al. Phase II study of preoperative bevacizumab, capecitabine and radiotherapy for resectable locally-advanced rectal cancer. Companion Pap: In press.

13. Batchelor TT, Sorensen AG, di Tomaso E, Zhang W-T, Duda DG, Cohen KS, et al. AZD2171, a pan-VEGF receptor tyrosine kinase inhibitor, normalizes tumor vasculature and alleviates edema in glioblastoma patients. Cancer Cell. 2007;11:83-95.

14. Goede V, Coutelle O, Neuneier J, Reinacher-Schick A, Schnell R, Koslowsky TC, et al. Identification of serum angiopoietin-2 as a biomarker for clinical outcome of colorectal cancer patients treated with bevacizumab-containing therapy. Br J Cancer. 2010;103:1407-14.

15. Kaseb AO, Garrett-Mayer E, Morris JS, Xiao L, Lin E, Onicescu G, et al. Efficacy of bevacizumab plus erlotinib for advanced hepatocellular carcinoma and predictors of outcome: final results of a phase II trial. Oncology. 2012;82:67-74.

16. Loupakis F, Falcone A, Masi G, Fioravanti A, Kerbel RS, Del Tacca M, et al. Vascular endothelial growth factor levels in immunodepleted plasma of cancer patients as a possible pharmacodynamic marker for bevacizumab activity. J Clin Oncol Off J Am Soc Clin Oncol. 2007;25:1816-8. 
17. Lu J-F, Bruno R, Eppler S, Novotny W, Lum B, Gaudreault J. Clinical pharmacokinetics of bevacizumab in patients with solid tumors. Cancer Chemother Pharmacol. 2008;62:779-86.

18. Willett CG, Boucher Y, Duda DG, di Tomaso E, Munn LL, Tong RT, et al. Surrogate markers for antiangiogenic therapy and dose-limiting toxicities for bevacizumab with radiation and chemotherapy: continued experience of a phase I trial in rectal cancer patients. J Clin Oncol Off J Am Soc Clin Oncol. 2005;23:8136-9.

19. Zahiragic L, Schliemann C, Bieker R, Thoennissen NH, Burow K, Kramer C, et al. Bevacizumab reduces VEGF expression in patients with relapsed and refractory acute myeloid leukemia without clinical antileukemic activity. Leukemia. 2007;21:1310-2.

20. Zhu AX, Sahani DV, Duda DG, di Tomaso E, Ancukiewicz M, Catalano OA, et al. Efficacy, safety, and potential biomarkers of sunitinib monotherapy in advanced hepatocellular carcinoma: a phase II study. J Clin Oncol Off J Am Soc Clin Oncol. 2009;27:3027-35.

21. Harmey JH, Bouchier-Hayes D. Vascular endothelial growth factor (VEGF), a survival factor for tumour cells: implications for anti-angiogenic therapy. BioEssays News Rev Mol Cell Dev Biol. 2002;24:280-3.

22. Saclarides TJ, Speziale NJ, Drab E, Szeluga DJ, Rubin DB. Tumor angiogenesis and rectal carcinoma. Dis Colon Rectum. 1994;37:921-6.

23. Takebayashi Y, Aklyama S, Yamada K, Akiba S, Aikou T. Angiogenesis as an unfavorable prognostic factor in human colorectal carcinoma. Cancer. 1996;78:226-31.

24. Tanigawa N, Amaya H, Matsumura M, Lu C, Kitaoka A, Matsuyama K, et al. Tumor angiogenesis and mode of metastasis in patients with colorectal cancer. Cancer Res. 1997:57:1043-6.

25. Tomisaki S, Ohno S, Ichiyoshi Y, Kuwano H, Maehara Y, Sugimachi K. Microvessel quantification and its possible relation with liver metastasis in colorectal cancer. Cancer. 1996;77(8 Suppl):1722-8.

26. Mandard AM, Dalibard F, Mandard JC, Marnay J, Henry-Amar M, Petiot JF, et al. Pathologic assessment of tumor regression after preoperative chemoradiotherapy of esophageal carcinoma. Clinicopathologic correlations Cancer. 1994;73:2680-6.

27. Weidner N. Current pathologic methods for measuring intratumoral microvessel density within breast carcinoma and other solid tumors. Breast Cancer Res Treat. 1995;36:169-80.

28. Bazarbashi S, El-Bassiouni M, Abdelsalam M, Soudy H, Sanea NA, Jabbar AA et al. A modern regimen of pre-operative concurrent chemo-radiation therapy in locally advanced rectal cancer. J Surg Oncol. 2008;98:167-74.

29. Chan AK, Wong AO, Jenken DA. Preoperative capecitabine and pelvic radiation in locally advanced rectal cancer-is it equivalent to 5-FU infusion plus leucovorin and radiotherapy? Int J Radiat Oncol Biol Phys. 2010;76:1413-9.

30. Craven I, Crellin A, Cooper R, Melcher A, Byrne P, Sebag-Montefiore D. Preoperative radiotherapy combined with 5 days per week capecitabine chemotherapy in locally advanced rectal cancer. $\mathrm{Br} J$ Cancer. 2007;97:1333-7.

31. De Bruin AFJ, Nuyttens JJ, Ferenschild FTJ, Planting AST, Verhoef C, de Wilt $J H W$. Preoperative chemoradiation with capecitabine in locally advanced rectal cancer. Neth J Med. 2008;66:71-6.

32. Dupuis O, Vie B, Lledo G, Hennequin C, Noirclerc M, Bennamoun M, et al. Preoperative treatment combining capecitabine with radiation therapy in rectal cancer: a GERCOR Phase II Study. Oncology. 2007;73:169-76.

33. Kim J-S, Kim J-S, Cho M-J, Song K-S, Yoon W-H. Preoperative chemoradiation using oral capecitabine in locally advanced rectal cancer. Int J Radiat Oncol Biol Phys. 2002;54:403-8

34. Korkolis DP, Boskos CS, Plataniotis GD, Gontikakis E, Karaitianos IJ, Avgerinos $\mathrm{K}$, et al. Pre-operative chemoradiotherapy with oral capecitabine in locally advanced, resectable rectal cancer. Anticancer Res. 2007;27:541-5.

35. Marsh RW, George TJ, Siddiqui T, Mendenhall WM, Zlotecki RA, Grobmyer S, et al. A phase II trial of neoadjuvant capecitabine combined with hyperfractionated accelerated radiation therapy in locally advanced rectal cancer. Am J Clin Oncol. 2010;33:251-6.

36. Simon R, Wittes RE, Ellenberg SS. Randomized phase II clinical trials. Cancer Treat Rep. 1985;69:1375-81.

37. McShane LM, Altman DG, Sauerbrei W, Taube SE, Gion M, Clark GM. Reporting Recommendations for Tumor Marker Prognostic Studies. J Clin Oncol. 2005;23:9067-72.

38. Aschele C, Cionini L, Lonardi S, Pinto C, Cordio S, Rosati G, et al. Primary tumor response to preoperative chemoradiation with or without oxaliplatin in locally advanced rectal cancer: pathologic results of the STAR-01 randomized phase III trial. J Clin Oncol Off J Am Soc Clin Oncol. 2011;29:2773-80

39. Gérard J-P, Azria D, Gourgou-Bourgade S, Martel-Laffay I, Hennequin C, Etienne $P$ - $L$, et al. Comparison of two neoadjuvant chemoradiotherapy regimens for locally advanced rectal cancer: results of the phase III trial ACCORD 12/0405-Prodige 2. J Clin Oncol Off J Am Soc Clin Oncol. 2010;28:1638-44

40. Chua YJ. Pathological complete response: still a relevant endpoint in rectal cancer? Lancet Oncol. 2010;11:807-8.

41. Jubb AM, Harris AL. Biomarkers to predict the clinical efficacy of bevacizumab in cancer. Lancet Oncol. 2010;11:1172-83.

42. Augustin HG, Koh GY, Thurston G, Alitalo K. Control of vascular morphogenesis and homeostasis through the angiopoietin-Tie system. Nat Rev Mol Cell Biol. 2009;10:165-77.

43. Hanahan D. Signaling vascular morphogenesis and maintenance. Science. 1997;277:48-50

44. Imanishi Y, Hu B, Jarzynka MJ, Guo P, Elishaev E, Bar-Joseph I, et al Angiopoietin-2 stimulates breast cancer metastasis through the alpha(5) beta(1) integrin-mediated pathway. Cancer Res. 2007;67:4254-63.

45. Jubb AM, Hurwitz HI, Bai W, Holmgren EB, Tobin P, Guerrero AS, et al. Impact of vascular endothelial growth factor-A expression, thrombospondin-2 expression, and microvessel density on the treatment effect of bevacizumab in metastatic colorectal cancer. J Clin Oncol Off J Am Soc Clin Oncol. 2006;24:217-27

\section{Submit your next manuscript to BioMed Central and take full advantage of:}

- Convenient online submission

- Thorough peer review

- No space constraints or color figure charges

- Immediate publication on acceptance

- Inclusion in PubMed, CAS, Scopus and Google Scholar

- Research which is freely available for redistribution 